\title{
Pregnancy and fetal outcomes of symptoms of sleep-disordered breathing
}

\author{
G. Bourjeily*, C.A. Raker ${ }^{\#}$, M. Chalhoub and M.A. Miller ${ }^{+}$
}

ABSTRACT: The physiological changes of pregnancy may predispose females to develop sleepdisordered breathing (SDB) or protect against it. Studies evaluating outcomes of SDB symptoms in pregnancy are scarce. The goal of this study was to evaluate the prevalence of SDB symptoms in pregnancy and their relationship with pregnancy and neonatal outcomes.

A cross-sectional survey of randomly selected immediate postpartum females was performed using the multivariable apnoea prediction index. Record review, including demographics and medical history, was performed. Main outcome measures included pregnancy and neonatal outcomes.

1,000 subjects were recruited. Mean \pm SD age was $29.1 \pm 6.1$ yrs. Factors used in the regression analysis included age, body mass index, diabetes, chronic hypertension, multifetal gestations, smoking and renal disease. Snoring was present in $35.1 \%$ of subjects. Symptoms of SDB were associated with a higher likelihood of pregnancy-induced hypertension and pre-eclampsia (adjusted OR 2.3, 95\% Cl 1.4-4.0), gestational diabetes (adjusted OR 2.1, 95\% Cl 1.3-3.4) and unplanned Caesarean deliveries (adjusted OR 2.1, 95\% $\mathrm{Cl}$ 1.4-3.2) after multivariable regression analysis. Gasping may have been associated with a higher likelihood of preterm delivery, after adjusting for age and multifetal pregnancies (adjusted OR 1.8, 95\% $\mathrm{Cl} 1.1-3.2$ ) but this association appeared to be mediated by pre-eclampsia.

Symptoms of SDB are common in pregnancy and associated with a higher likelihood of gestational hypertensive disorders, gestational diabetes and unplanned Caesarean deliveries.

KEYWORDS: Diabetes, fetal outcomes, gestational, hypertension, pregnant females, snoring

S leep-disordered breathing (SDB) occurs in $9 \%$ of young-middle aged females [1] and $56 \%$ of older females [2]. Females report snoring less commonly than males and have significant differences in sleep latency, sleep architecture, prevalence of obesity, and predictive power of certain anthropometric features [3].

SDB is associated with adverse cardiovascular outcomes in females. Snoring has been associated with higher risk of elevated glycosylated haemoglobin in premenopausal females, [4] and obstructive sleep apnoea (OSA) is linked to decreased insulin sensitivity [5]. Associations with the metabolic syndrome have been described [6, 7], especially in females with severe OSA [7]. Despite the controversy in the development of new onset hypertension in patients with OSA in the Wisconsin Sleep Cohort Study [8] and the Sleep Heart Health Study [9], the association of OSA with hypertension was described in females [8, 10, 11]. In a recent study, the relative risk of hypertension in subjects with OSA was higher than controls (apnoea/hypopnoea index <15) and similar in males and females [11]. Another study reported that normotensive females snoring occasionally or regularly have a $29 \%$ and $55 \%$ increased risk of developing hypertension, respectively, compared with non-snorers [10].

Many physiological changes that predispose to the development of SDB occur in pregnancy [12]. Although studies have reported that snoring occurs in $14-45 \%$ of pregnant females [13-15], the incidence of SDB remains unknown. A few studies suggest a significant association between habitual snoring and gestational hypertensive disorders [16]. Others have assessed a potential association with neonatal outcomes [14, 17], with some showing growth restriction in snorers compared with non-snorers [14] and others failing to show a correlation [17]. Despite an association

Earn CME accreditation by answering questions about this article. You will find these at the back of the printed copy of this issue or online at www.erj.ersjournals.com/cmeinfo.dtl

AFFILIATIONS

*Pulmonary and Critical Care Medicine, Dept of Medicine, ${ }^{+}$Division of Obstetric Medicine, Dept of Medicine,

"Division of Research, Dept of Obstetrics and Gynecology, Women and Infants Hospital of Rhode Island, Providence, RI, and

'Dept of Pulmonary Medicine, Staten Island University Hospital, Staten Island, NY, USA.

CORRESPONDENCE

G. Bourjeily

Dept of Medicine

Women and Infants Hospital of

Rhode Island

100 Dudley Street

3rd Floor

Providence

RI 02905

USA

E-mail: GBourjeily@wihri.org

Received:

Feb 092010

Accepted after revision:

April 192010

First published online:

June 042010 
between SDB, glucose intolerance and insulin resistance outside pregnancy $[18,19]$, no studies have evaluated a link in pregnancy. In addition, there are no studies evaluating an association between SDB symptoms and mode of delivery, although a potential effect of total sleep duration on delivery mode has been described in some studies [20] but not in others [21].

The aim of this study was to determine the incidence of SDB symptoms in pregnancy in a large cohort of patients, and to assess potential associations between SDB symptoms and cardiovascular and obstetric maternal outcomes, as well as neonatal birth outcomes.

\section{METHODS}

\section{Subjects and protocol}

\section{Female subjects}

The study protocol was approved by the Institutional Review Board (Women and Infants Hospital of RI, Providence, RI, USA) and consent was obtained. Subjects were selected randomly from daily lists of all deliveries and recruited at a tertiary care obstetric hospital over 18 months. English-speaking patients $\geqslant 18$ yrs old were surveyed $24-48 \mathrm{~h}$ postpartum, with or without a bed partner. Deliveries with fetal or neonatal demise were excluded.

The questionnaire elicited information regarding demographics, medical history, sleep-related symptoms, medications, and pregnancy-related conditions. Neck circumference was measured at the time of consent. Patients' records were reviewed to corroborate patient-reported outcomes and any discrepancies between sources were resolved by the principal investigator. Pre-eclampsia was classified based on the American College of Obstetricians and Gynecologists' definition [22]. The definition of gestational diabetes was based on CARPENTER and COUSTAN's criteria [23], and the American Diabetes Association [24]. Mode of delivery was vaginal, planned Caesarean delivery or unplanned Caesarean delivery.

SDB symptoms in the 3 months preceding delivery were assessed using index 1 of the Multivariable Apnoea Prediction Index (MAPI) [25]. Questions read as follows. In the last 3 months of your pregnancy, how often have you experienced (or were you told) about the following symptoms? 1) You snored loudly; 2)You snorted or gasped; 3)Your breathing stopped, you choked or you struggled for breath. Answers: 0) Never; 1) Rarely (less than once a week); 2) Sometimes (1-2 times a week); 3) Frequently (3-4 times a week); 4) Always (5-7 times a week).

MAPI was calculated using both pre-pregnancy body mass index (BMI) obtained at the time of the first prenatal visit and BMI at delivery and the two results were compared but not correlated with outcomes since this tool has not been validated in pregnancy.

2 weeks following delivery, patients were surveyed by telephone regarding interim complications.

\section{Newborn subjects}

Outcomes evaluated included weight for gestational age, and 1 and 5-min Apgar scores. Growth restriction was defined based on data by ALEXANDER et al. [26] on normal fetal growth based on gestational age. Low birth weight was defined as $<2,500 \mathrm{~g}$ and growth restriction as $<10$ th percentile for gestational age.

\section{Statistical analysis}

Data analysis was performed with SAS version 9.1 (SAS Institute, Cary, NC, USA). SDB symptom frequencies were categorised as never/rarely, sometimes, or frequently/always. Unsure or missing responses were excluded.

Categorical variables were compared by Fisher's exact test and continuous variables were compared between groups by ANOVA. Continuous variables were examined for deviations from normality and homoskedasticity prior to ANOVA. Twotailed p-values were reported, with $\mathrm{p}<0.05$ considered statistically significant.

Unconditional multivariable logistic regression was used to estimate adjusted OR (aOR) and 95\% CI for the association of SDB symptom frequency and pregnancy outcomes. Factors used in the regression analysis included age, BMI, diabetes mellitus, chronic hypertension, multifetal gestations, renal disease, and cigarette smoking. Because pre-eclampsia- and pregnancy-induced hypertension were uncommon in the study cohort, they were analysed together as one outcome. Delivery method was examined by multinomial logistic regression with successful vaginal delivery as the referent. Preterm delivery $(<37$ weeks gestational age) and neonatal outcomes were examined by logistic regression. Robust variance estimates were obtained for neonatal outcomes to account for correlation within multiple births. For all models, continuous variables were entered as linear covariates, since testing of polynomial terms did not suggest nonlinearity. Model fit was checked by the Hosmer-Lemeshow test (logistic regression) or inspection of residuals.

\section{RESULTS}

1,000 females agreed to participate in the study with a rate of decline $<3 \%$. Patient demographics and clinical characteristics are detailed in table 1 . Over $22 \%$ had a pre-pregnancy BMI $\geqslant 30$. Bed partners were present at the time of interview for $36 \%$ of the patients and $78 \%$ of patients reported having a bed partner.

\section{Symptoms of SDB}

Loud snoring occurred frequently/always in 35\% of subjects (table 2), and $37 \%$ reported at least one symptom. There was a trend toward statistical significance in snoring frequency $(p=0.05)$ between the groups with and without a bed partner present in the room. However, the presence of the bed partner during the interview did not affect symptom frequency among patients with a bed partner. Greater symptom frequency was associated with neck circumference (fig. 1) and older age but not smoking. Snoring frequency was significantly correlated with pre-pregnancy BMI, delivery BMI, and pregnancy weight gain (fig. 1).

MAPI was calculated using both BMI values. As expected, the MAPI predicting $\geqslant 50 \%$ likelihood of SDB was lower using pre-pregnancy BMI. However, the sensitivity of frequently/ always snoring, gasping and choking for MAPI $\geqslant 50 \%$ was higher using pre-pregnancy BMI. These findings suggest that pre-pregnancy obesity may be a more important risk factor than pregnancy weight gain. 


\section{TABLE 1 Demographics and clinical characteristics}

\begin{tabular}{lc} 
Age yrs & $29.1 \pm 6.1$ \\
Race/ethnicity n & 984 \\
White, non-Hispanic \% & 69 \\
Black, non-Hispanic \% & 10 \\
Hispanic \% & 16 \\
Asian \% & 3 \\
Other \% & 2 \\
BMI & \\
Pre-pregnancy & $26.1 \pm 6.2$ \\
At delivery & $32.1 \pm 6.3$ \\
Weight gain lbs & $34.7 \pm 16.2$ \\
Neck circumference cm & $36 \pm 2.7$ \\
Cigarette smoking n & 998 \\
Nonsmoker \% & 76 \\
Prior to pregnancy \% & 14 \\
Current smoker \% & 9 \\
Medical conditions & \\
DM, type I or II \% & 0.7 \\
Gestational diabetes \% & 10 \\
Pregnancy induced hypertension, current or prior & 11 \\
pregnancy \% & \\
Pre-eclampsia, current or prior pregnancy & 8 \\
Multi-gestational pregnancy \% & 2.5 \\
Delivery method $\mathbf{n}$ & 1000 \\
Vaginal \% & 53 \\
Cesarean section \% & 33 \\
Vaginal to Cesarean section \% & 14 \\
Birth weight (total) g & $3265 \pm 667$ \\
Singles & $3312 \pm 635$ \\
Multiples & $2345 \pm 611$ \\
Low birth weight (<2500 g) \% & 9.9 \\
Small for gestational age (total) \% & 3.4 \\
Singles \% & 2.9 \\
Multiples \% & 12.5 \\
& \\
\hline
\end{tabular}

Data are presented as mean $\pm \mathrm{SD}$, unless otherwise stated. BMI: body mass index DM: diabetes mellitus. Percentages may not sum to $100 \%$ due to rounding.

\section{Symptoms of SDB and pregnancy outcomes}

Snoring significantly correlated with current pregnancy-induced hypertension and pre-eclampsia. The association remained significant even after adjusting for multiple factors (table 3).

Snoring and gasping were associated with gestational diabetes and this association remained significant in a multivariable regression analysis (table 3 ). When all three symptoms were combined, the association with gestational diabetes was stronger (OR 6.1, 95\% CI 2.3-16.2) even when adjusted for the other factors (aOR 4.0, 95\% 1.4-11.1).

\section{Delivery outcomes}

Delivery rates are detailed in table 1 . Although the rate of Caesarean sections was higher than that of the general population, the association between SDB symptoms and pregnancyrelated conditions in the Caesarean section group were otherwise similar to the vaginal delivery group. There was a significantly higher prevalence of Caesarean sections in patients with loud snoring and gasping even after adjusting for multifetal gestations,

\section{TABLE 2 Sleep-disordered breathing (SDB) symptom frequency and patient age ${ }^{\#}$}

\begin{tabular}{lccc} 
SDB symptom & Subjects n (\%) & Mean \pm SD & p-value \\
\hline Snoring loudly & & & \\
$\quad$ Never/rarely & $483(51)$ & $28.2 \pm 6.0$ & \\
$\quad$ Sometimes & $133(14)$ & $29.6 \pm 5.9$ & \\
$\quad$ Frequently/always & $333(35)$ & $30.3 \pm 6.0$ & \\
Gasping/snorting & & & $<0.0001$ \\
$\quad$ Never/rarely & $777(79)$ & $28.6 \pm 6.1$ & \\
$\quad$ Sometimes & $101(10)$ & $30.8 \pm 6.3$ & \\
$\quad$ Frequently/always & $104(11)$ & $30.5 \pm 5.4$ & \\
Choking/stopped breathing & & & 0.02 \\
$\quad$ Never/rarely & $891(90)$ & $29.0 \pm 6.1$ & \\
$\quad$ Sometimes & $55(6)$ & $28.6 \pm 5.7$ & \\
$\quad$ Frequently/always & $48(5)$ & $31.4 \pm 6.8$ & \\
& & & \\
& &
\end{tabular}

Percentages may not sum to $100 \%$ due to rounding. "Unsure" responses for snoring ( $n=51)$, gasping $(n=18)$, and choking $(n=6)$ were omitted. $\#: n=1,000$

smoking and fetal weight (table 4). However, when the statistical model was further adjusted for pre-eclampsia, pregnancyinduced hypertension, gestational diabetes, and BMI at delivery, only the association with unplanned Caesarean sections remained significant for both snoring (aOR 1.7, 95\% CI 1.1-2.6) and gasping (aOR 3.6, 95\% CI 2.0-6.4).

Mean gestational age at birth was $38.6 \pm 2.4$ weeks, and $12 \%$ of births were preterm (delivery before 37 weeks gestational age). Gasping was associated with a significantly higher likelihood of preterm delivery, even after adjusting for age, smoking and multifetal pregnancies (aOR 1.9, 95\% CI 1.1-3.3).

\section{Fetal outcomes}

When birth weight for gestational age was assessed, there was a trend towards an association between snoring and growth restriction (OR 1.9, 95\% CI 0.8-4.3) but the outcomes were too few for a multivariable logistic regression analysis. Gasping was significantly associated with Apgar scores at 1 min (fig. 2) even after adjusting for multifetal pregnancy, smoking and maternal age (aOR 1.9, 95\% CI 1.0-3.4), but not after adjusting for pre-eclampsia. However, $>98 \%$ of 5-min Apgar scores were $\geqslant 7$. For that reason, although 5-min scores appear significant (aOR 3.7, 95\% CI 1.1-11.9), this may be due to bias and small numbers.

\section{Follow-up questionnaire}

A total of 227 patients were not available for follow-up. When compared to patients who were available, those lost to followup were 2 yrs older and more likely to be Hispanic, but otherwise showed no difference in outcomes studied. Of those $227,16(7 \%)$ had returned to the emergency room within 2 weeks of delivery, most commonly for hypertension. Since this record review would not identify all patients with postpartum hypertension, this group was excluded from further follow-up analysis.

Of the 773 patients who completed follow-up, $5.4 \%$ had delivery complications and $6.2 \%$ returned to the hospital. These 


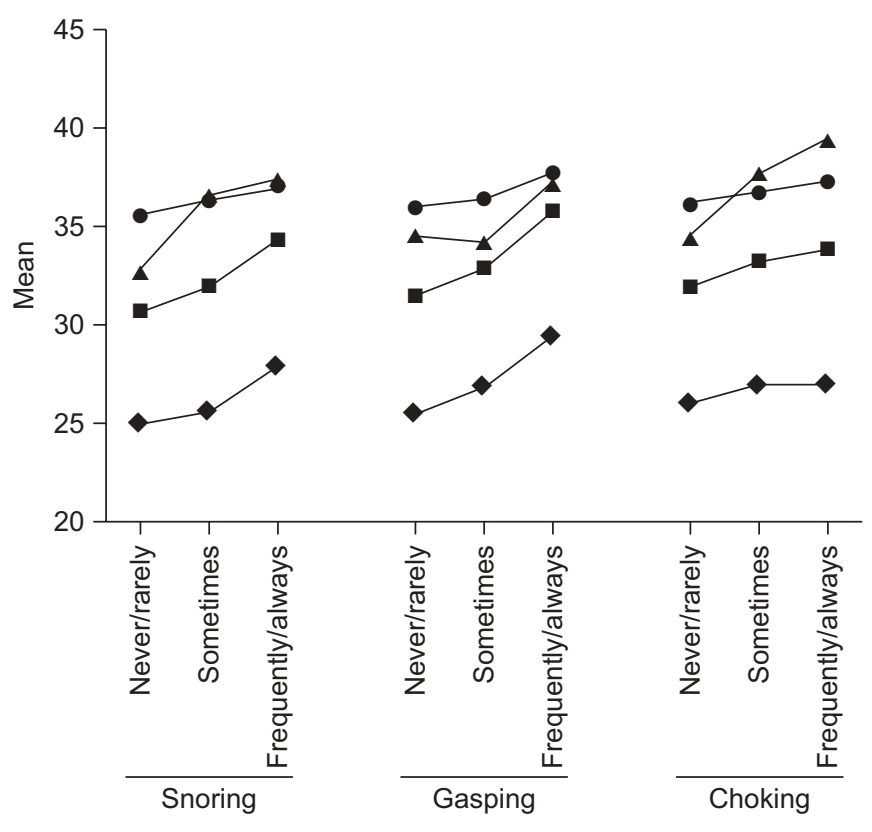

FIGURE 1. Frequency of sleep-disordered breathing symptoms and mean neck circumference, weight gain, and body mass index (BMI). - neck circumference $\mathrm{cm} ; \mathbf{\Lambda}$ : weight gain lbs; $\mathbf{\square}$ : BMl at delivery; $\bullet$ : BMl pre-pregnancy. $p<0.05$ for all comparisons except for choking and BMl pre-pregnancy $(p=0.3)$ and at delivery $(p=0.05)$ and gasping and weight gain $(p=0.3)$.

complications were significantly associated with gasping (OR 3.0, 95\% CI 1.4-6.7, and 2.4, 95\% CI 1.3-4.6, respectively) and choking (OR 4.0, 95\% CI 1.4-11.1, and 3.7, 95\% CI 1.6-8.3, respectively). Hypertension or pre-eclampsia were the most common postpartum conditions reported (13.1\%). After excluding patients with all types of antenatal hypertension, there was a significant association between SDB symptoms and the development of postpartum hypertension in the 2 weeks following delivery (OR 2.6, 95\% CI 1.2-5.5, for snoring).

\section{DISCUSSION}

Symptoms of SDB were common in our randomly selected cross-sectional evaluation of immediate postpartum pregnant females. Our results are consistent with other studies showing a prevalence of snoring in $14-45 \%$ of pregnant females [14, 15, 27]. Although having a bed partner was thought to result in more frequent reporting of SDB symptoms among pregnant females, our study did not substantiate this theory. Snoring is a good predictor of SDB in nonpregnant patients; however, the predictive power of this and other symptoms in pregnancy is not well established and may be lower. In a small study by SAHIN et al. [28], only four out of 35 (11.4\%) pregnant females suspected of having OSA based on the Berlin questionnaire were found to have OSA on polysomnography, suggesting a suboptimal positive predictive value of the questionnaire in this patient population. Snoring could be indicative of flow limitation, for instance, which would not meet criteria for apnoea or hypopnoea. A recent study has found a significantly elevated apnoea/hypopnoea index, with hypopnoeas defined as a flow limitation without desaturation in females with gestational hypertension; however, the study did not evaluate the association of these findings with symptoms of SDB [29]. Evidence evaluating the association of symptoms of SDB with polysomnographic evidence of SDB in pregnancy is lacking. Therefore, the higher prevalence of symptoms in pregnancy does not necessarily portend a higher incidence of SDB.

\begin{tabular}{|c|c|c|c|c|c|c|c|}
\hline \multirow[t]{3}{*}{ Symptom } & \multirow[t]{3}{*}{ Total } & \multicolumn{3}{|c|}{ Hypertension and/or pre-eclampsia } & \multicolumn{3}{|c|}{ Gestational diabetes } \\
\hline & & \multirow[t]{2}{*}{ n (\%) } & \multicolumn{2}{|c|}{ OR $(95 \% \mathrm{Cl})$} & \multirow[t]{2}{*}{ n (\%) } & \multicolumn{2}{|c|}{ OR $(95 \% \mathrm{Cl})$} \\
\hline & & & Unadjusted & Adjusted & & Unadjusted & Adjusted $^{+}$ \\
\hline \multicolumn{8}{|l|}{ Snoring loudly } \\
\hline Never/rarely & 480 & $25(5)$ & 1.0 Ref. & 1.0 Ref. & $31(6)$ & 1.0 Ref. & 1.0 Ref. \\
\hline Sometimes & 133 & $14(11)$ & $2.1(1.1-4.3)$ & $1.7(0.8-3.5)$ & $11(8)$ & $1.3(0.6-2.7)$ & $1.2(0.6-2.4)$ \\
\hline Frequently/always & 331 & $59(18)$ & $4.0(2.4-6.5)$ & $2.3(1.4-4.0)$ & $52(16)$ & $2.7(1.7-4.3)$ & $2.1(1.3-3.4)$ \\
\hline \multicolumn{8}{|l|}{ Gasping/snorting } \\
\hline Never/rarely & 775 & $61(8)$ & 1.0 Ref. & 1.0 Ref. & $60(8)$ & 1.0 Ref. & 1.0 Ref. \\
\hline Sometimes & 55 & $9(16)$ & $1.8(0.9-3.9)$ & $1.6(0.7-3.6)$ & $6(11)$ & $1.2(0.5-2.9)$ & $1.2(0.5-3.0)$ \\
\hline Frequently/always & 48 & $6(13)$ & $1.3(0.6-3.2)$ & $0.9(0.3-2.4)$ & $10(21)$ & $2.6(1.3-5.5)$ & $2.0(0.9-4.3)$ \\
\hline
\end{tabular}


TABLE 4 Association of sleep-disordered breathing symptoms with method of delivery

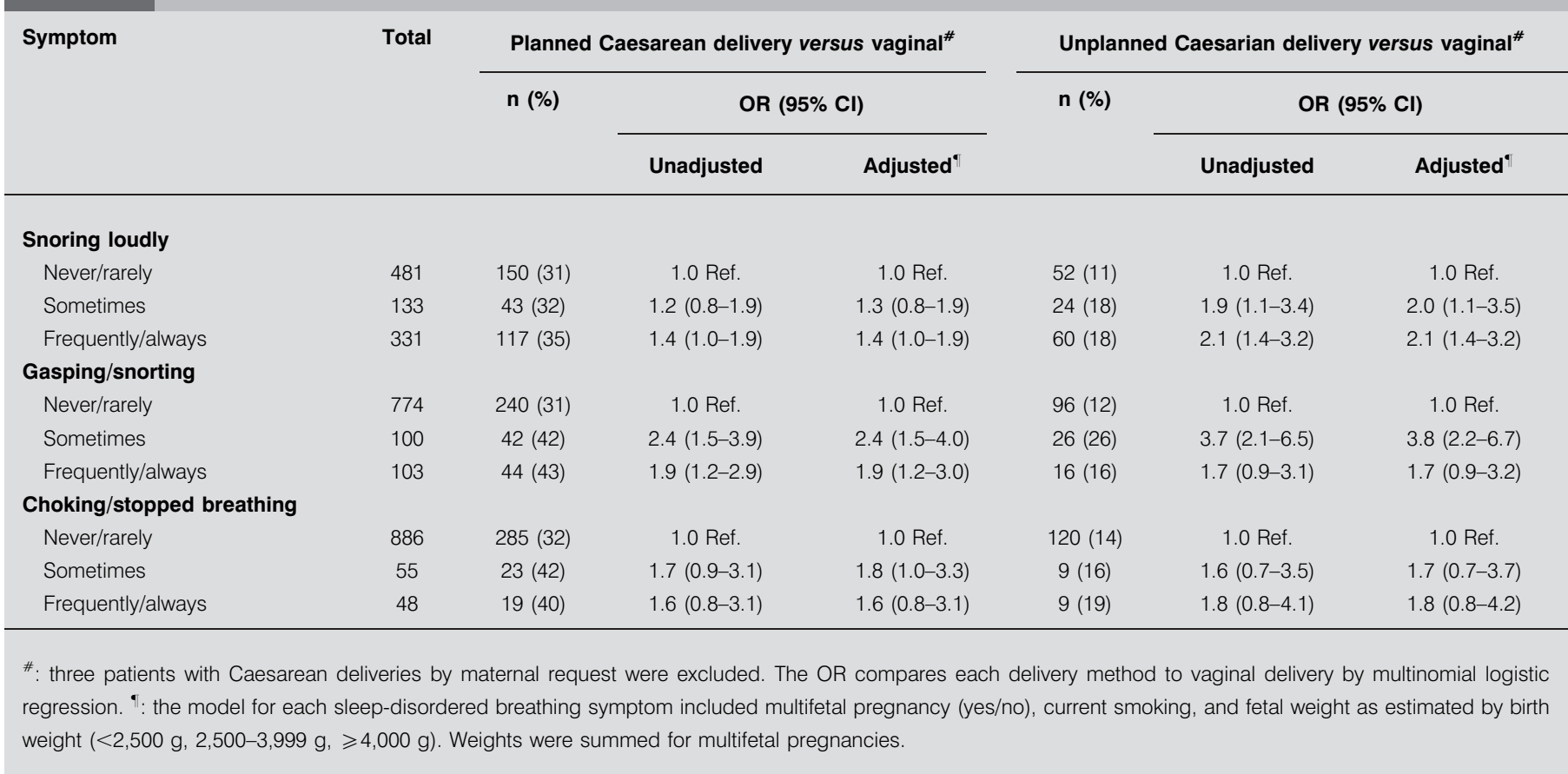

In our sample population, about $22 \%$ of subjects met criteria for obesity (BMI >30), a lower prevalence than the National Health and Nutrition Examination Survey [30] for adult females, suggesting that our population may be less overweight than the general population, potentially underestimating the incidence of SDB symptoms.

\section{SDB symptoms and gestational hypertensive disorders}

Our study shows a significant correlation between SDB symptoms and gestational hypertensive disorders, even after correcting for major risk factors, and is consistent with prior reports $[13,14,16,31]$. The association in this study could have been negatively affected by our definition of pre-eclampsia [22], which was more conservative than the one recently

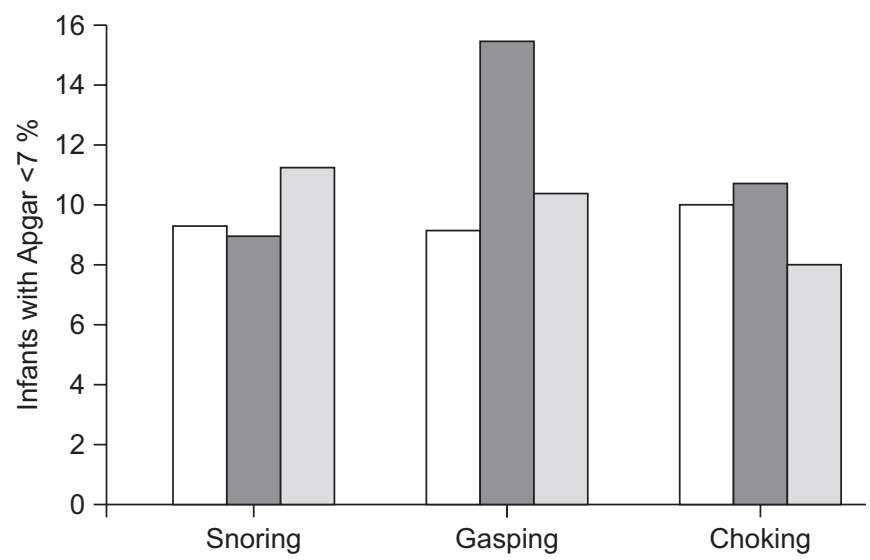

FIGURE 2. Sleep-disordered breathing symptoms and 1-min Apgar scores. $\square$ : never/rarely; $\mathbf{a}:$ sometimes; $\quad$ : frequently/always. Gasping sometimes versus never or rarely was associated with Apgar $1-\min <7$ (15.4\% versus 9.1\%; $p=0.04)$. All other comparisons were nonsignificant. outlined by the Canadian Society of Obstetrics and Gynecology [32]; regardless, the association remained significant.

The underlying mechanism for the association of SDB with pregnancy-induced hypertension and pre-eclampsia has not been elucidated. Hypoxaemia may be a potential contributor. Data from chronically hypoxic high-altitude residents strongly suggest higher rates of pre-eclampsia and fetal growth restriction compared to low-altitude residents [33, 34]. Data on intermittent hypoxia and pregnancy outcomes are scarce, but intermittent hypoxia may lead to endothelial dysfunction, which is present in patients with OSA in all severity categories $[35,36]$. Endothelial dysfunction and angiogenic-antiangiogenic disequilibrium have been implicated as a possible underlying mechanism in pre-eclampsia. A recent study has tied both disorders with endothelial dysfunction and showed a significant association between pre-eclampsia, OSA and endothelial dysfunction in pregnant women [37]. These data suggest intermittent hypoxia in OSA as a potential link leading to placental hypoxaemia, triggering a cascade of events resulting in pre-eclampsia.

It is also possible that flow limitations play a mechanistic role in the development of gestational hypertensive disorders. Frequent, prolonged flow limitations without desaturations have been identified in patients with pre-eclampsia [38] or with risk factors for pre-eclampsia [39]. Treatment of patients with risk factors for pre-eclampsia and flow limitations (without SDB) with continuous positive airway pressure (CPAP) throughout pregnancy has favourable effects on blood pressure $[39,40]$ and fetal outcomes compared to controls [40]. Although these findings indirectly suggest that flow limitation may have a causative role in blood pressure control in pregnancy, the numbers are quite small, hence the need for larger studies. Moreover, nocturnal CPAP treatment for one 
night increases cardiac output [41] and improves blood pressure control [42] in patients with pre-eclampsia, suggesting several potential beneficial effects. Therefore, it is possible that even in the absence of hypoxia and re-oxygenation, haemodynamic changes associated with flow limitations may be implicated in the pathogenesis of pre-eclampsia in patients with SDB symptoms but without SDB.

Another possibility is that pre-eclampsia predisposes to the development of SDB. Patients with pre-eclampsia have lower oncotic pressures than normal pregnant females, a larger neck circumference and a smaller upper airway [43]. Additional studies are needed to evaluate this theory further.

\section{Gestational diabetes}

Our study is the first to examine the association of gestational diabetes and obstetric outcomes with symptoms of SDB. There was a significant association between SDB symptoms and gestational diabetes even after controlling for BMI at delivery, suggesting a potentially different mechanism than obesityrelated insulin resistance. The association with gestational diabetes was even stronger when all three symptoms were combined, suggesting that gestational diabetes is more likely in patients with a higher likelihood of SDB, rather than in those with an isolated symptom. Although no prior studies have suggested this association in pregnancy, data from the nonpregnant population suggest an association between SDB, insulin resistance and glucose intolerance [18, 19, 44, 45]. The mechanism behind this association in pregnancy that is independent of BMI needs to be elucidated further.

\section{Mode of delivery}

Our study has also shown that unplanned Caesarean deliveries were more likely to occur in snorers compared to non-snorers, even after adjusting for potential confounders, suggesting that snoring may be an independent predictor of unplanned Caesarean sections. Although existing data suggest that obesity is associated with higher rates of Caesarean sections [46], the mechanism in our study seemed to be independent of BMI. A possible hypothesis to explain this association may be that anthropometric measures associated with failed vaginal deliveries may also be associated with SDB.

The effects of sleep duration on labour and delivery outcomes have been inconsistent $[20,21]$. While the study of EvANs et al. [20] showed no effect of sleep duration on delivery outcomes, studies using actigraphy showed that females with sleep deprivation at term had significantly longer labour and more Caesarean deliveries [21]. The link between sleep characteristics and the mode of delivery is not clear, but the association of symptoms of SDB with a higher BMI, gestational hypertensive disorders and gestational diabetes may in part explain higher rates of Caesarean sections. Another possibility would be whether sleep fragmentation is associated with adverse outcomes irrespective of SDB. Polysomnography may help elucidate the potential role of hypoxaemia and/or arousal indices.

\section{Neonatal outcomes}

A small number of studies have evaluated the association between SDB symptoms and fetal outcomes [14, 17, 29, 47] and are somewhat conflicting $[14,17]$ with some lacking a control arm [29]. A large study has shown an association between snoring and fetal growth restriction [14]. However, that study did not report comorbidities or adjust for confounders such as diabetes, hypertension and gestational hypertensive disorders. Our study did not show a significant association with growth restriction and is consistent with other studies [13, 17, 29, 48]. A limitation of most existing studies, including this study, relates to the lack of confirmation of gestational age by early gestation ultrasound in some subjects and the lack of longitudinal fetal growth data, leading to the possibility of missing a true effect. Future studies should take this factor into account. Our findings suggest that SDB symptoms may be associated with low 1-min Apgar scores, consistent with other studies [14], but not 5-min scores. However, 5-min scores are more predictive of future outcomes. Preterm birth seems to be associated with SDB symptoms; however, this association appears to be mediated by pre-eclampsia.

\section{Limitations of the study}

Our study is limited by the lack of a validated questionnaire for use in screening the pregnant population for SDB, and by a and lack of polysomnographic data. However, findings from our study suggest that, irrespective of the presence of SDB, the symptoms evaluated were common and did correlate with adverse outcomes. Future studies are needed that consider correlation of outcomes with polysomnography findings and validation of screening tools in this population.

\section{Conclusions}

In summary, our findings show that symptoms of SDB in pregnant females are associated with adverse pregnancy, delivery and fetal outcomes. Further studies are warranted to evaluate the mechanisms by which SDB causes these adverse outcomes, and to evaluate whether screening and treatment of SDB during pregnancy might improve outcomes.

\section{SUPPORT STATEMENT}

This study was supported by the Perkins Charitable Foundation.

\section{STATEMENT OF INTEREST}

None declared.

\section{ACKNOWLEDGEMENTS}

The authors would like to thank S.M. Martin, C. Citino, P. Auger and K. Connors (all Division of Research, Dept of Medicine, Women and Infants Hospital of Rhode Island, Providence, RI, USA) for their help with patient recruitment and data collection. These individuals were compensated for their contribution to the work. Perkins Charitable Foundation should also be acknowledged for their generous funding and support of the study. The authors are also grateful to: G. Oliva III and C. McPhee (both Springhill Syndications LLC, Los Angeles, CA, USA) for their help with obtaining funding; and G. Ankner and B. Hott (both Division of Research, Dept of Medicine, Women and Infants Hospital of Rhode Island) for their assistance with manuscript preparation.

\section{REFERENCES}

1 Douglas NJ. Clinicians' Guide to Sleep Medicine. London, Arnold, 2002.

2 Ancoli-Israel S, Kripke DF, Klauber MR, et al. Sleep-disordered breathing in community-dwelling elderly. Sleep 1991; 14: 486-995.

3 Lin CM, Davidson TM, Ancoli-Israel S. Gender differences in obstructive sleep apnea and treatment implications. Sleep Med Rev 2008; 12: 481-496. 
4 Joo S, Lee S, Choi HA, et al. Habitual snoring is associated with elevated hemoglobin A1c levels in non-obese middle-aged adults. J Sleep Res 2006; 15: 437-444.

5 Theorell-Haglow J, Berne C, Janson C, et al. Obstructive sleep apnoea is associated with decreased insulin sensitivity in females. Eur Respir J 2008; 31: 1054-1060.

6 Onat A, Hergenc G, Uyarel H, et al. Obstructive sleep apnea syndrome is associated with metabolic syndrome rather than insulin resistance. Sleep Breath 2007; 11: 23-30.

7 Sasanabe R, Banno K, Otake K, et al. Metabolic syndrome in Japanese patients with obstructive sleep apnea syndrome. Hypertens Res 2006; 29: 315-322.

8 Peppard PE, Young T, Palta M, et al. Prospective study of the association between sleep-disordered breathing and hypertension. N Engl J Med 2000; 342: 1378-1384.

9 O'Connor GT, Caffo B, Newman AB, et al. Prospective study of sleep-disordered breathing and hypertension: the Sleep Heart Health Study. Am J Respir Crit Care Med 2009; 179: 1159-1164.

$10 \mathrm{Hu}$ FB, Willett WC, Colditz GA, et al. Prospective study of snoring and risk of hypertension in women. Am J Epidemiol 1999; 150: 806-816.

11 Yukawa $\mathrm{K}$, Inoue $\mathrm{Y}$, Yagyu $\mathrm{H}$, et al. Gender differences in the clinical characteristics among Japanese patients with obstructive sleep apnea syndrome. Chest 2009; 135: 337-443.

12 Bourjeily G. Sleep disorders in pregnancy. Obstet Med 2009; 2 100-106.

13 Calaora-Tournadre D, Ragot S, Meurice JC, et al. [Obstructive sleep apnea syndrome during pregnancy: prevalence of main symptoms and relationship with pregnancy induced-hypertension and intrauterine growth retardation]. Rev Med Interne 2006; 27: 291-295.

14 Franklin KA, Holmgren PA, Jonsson F, et al. Snoring, pregnancyinduced hypertension, and growth retardation of the fetus. Chest 2000; 117: 137-141.

15 Schutte S, Del Conte A, Gross A, et al. Self-reported snoring and sleep in high risk pregnancies. Sleep Research 1995; 24: 342.

16 Ursavas A, Karadag M, Nalci N, et al. Self-reported snoring, maternal obesity and neck circumference as risk factors for pregnancy-induced hypertension and preeclampsia. Respiration 2008; 76: 33-39.

17 Loube DI, Poceta JS, Morales MC, et al. Self-reported snoring in pregnancy. Association with fetal outcome. Chest 1996; 109: 885-889.

18 Punjabi NM, Shahar E, Redline S, et al. Sleep-disordered breathing, glucose intolerance, and insulin resistance: the Sleep Heart Health Study. Am J Epidemiol 2004; 160: 521-530.

19 Punjabi NM, Sorkin JD, Katzel LI, et al. Sleep-disordered breathing and insulin resistance in middle-aged and overweight men. Am J Respir Crit Care Med 2002; 165: 677-682.

20 Evans ML, Dick MJ, Clark AS. Sleep during the week before labor: relationships to labor outcomes. Clin Nurs Res 1995; 4: 238-249.

21 Lee KA, Gay CL. Sleep in late pregnancy predicts length of labor and type of delivery. Am J Obstet Gynecol 2004; 191: 2041-2046.

22 ACOG practice bulletin. Diagnosis and management of preeclampsia and eclampsia. Number 33. Obstet Gynecol 2002; 99: 159-167.

23 Carpenter MW, Coustan DR. Criteria for screening tests for gestational diabetes. Am J Obstet Gynecol 1982; 144: 768-773.

24 American Diabetes Association. Diagnosis and classification of diabetes mellitus. Diabetes Care 2009; 32: Suppl. 1, S62-S67.

25 Maislin G, Pack AI, Kribbs NB, et al. A survey screen for prediction of apnea. Sleep 1995; 18: 158-166.

26 Alexander GR, Himes JH, Kaufman RB, et al. A United States national reference for fetal growth. Obstet Gynecol 1996; 87: 163-168.

27 Pien GW, Fife D, Pack AI, et al. Changes in symptoms of sleepdisordered breathing during pregnancy. Sleep 2005; 28: 1299-1305.
28 Sahin FK, Koken G, Cosar E, et al. Obstructive sleep apnea in pregnancy and fetal outcome. Int J Gynaecol Obstet 2008; 100: 141-146.

29 Champagne K, Schwartzman K, Opatrny L, et al. Obstructive sleep apnoea and its association with gestational hypertension. Eur Respir J 2009; 33: 559-565.

30 National Health and Nutrition Examination Survey (NHANES). www.cdc.gov/nchs/data/nhanes/databriefs/adultweight.pdf Date last accessed: February 2010. Date last updated: May 11, 2009

31 Perez-Chada D, Videla AJ, O'Flaherty ME, et al. Snoring, witnessed sleep apnoeas and pregnancy-induced hypertension. Acta Obstet Gynecol Scand 2007; 86: 788-792.

32 Magee LA, Helewa ME, Moutquin JM, et al. Diagnosis, evaluation, and management of the hypertensive disorders of pregnancy. J Obstet Gynecol Canada 2008; 30: S1-S48.

33 Jensen GM, Moore LG. The effect of high altitude and other risk factors on birthweight: independent or interactive effects? Am J Public Health 1997; 87: 1003-1007.

34 Miller S, Tudor C, Nyima, et al. Maternal and neonatal outcomes of hospital vaginal deliveries in Tibet. Int J Gynaecol Obstet 2007; 98: 217-221.

35 Jelic S, Padeletti M, Kawut SM, et al. Inflammation, oxidative stress, and repair capacity of the vascular endothelium in obstructive sleep apnea. Circulation 2008; 117: 2270-2278.

36 Kohler M, Craig S, Nicoll D, et al. Endothelial function and arterial stiffness in minimally symptomatic obstructive sleep apnea. Am J Respir Crit Care Med 2008; 178: 984-988.

37 Yinon D, Lowenstein L, Suraya S, et al. Pre-eclampsia is associated with sleep-disordered breathing and endothelial dysfunction. Eur Respir J 2006; 27: 328-333.

38 Connolly G, Razak AR, Hayanga A, et al. Inspiratory flow limitation during sleep in pre-eclampsia: comparison with normal pregnant and nonpregnant women. Eur Respir J 2001; 18: 672-676.

39 Guilleminault C, Palombini L, Poyares D, et al. Pre-eclampsia and nasal CPAP: Part 1. Early intervention with nasal CPAP in pregnant women with risk-factors for pre-eclampsia: preliminary findings. Sleep Med 2007; 9: 9-14.

40 Poyares D, Guilleminault C, Hachul H, et al. Pre-eclampsia and nasal CPAP: Part 2. Hypertension during pregnancy, chronic snoring, and early nasal CPAP intervention. Sleep Med 2007; 9: 15-21.

41 Blyton DM, Sullivan CE, Edwards N. Reduced nocturnal cardiac output associated with preeclampsia is minimized with the use of nocturnal nasal CPAP. Sleep 2004; 27: 79-84.

42 Edwards N, Blyton DM, Kirjavainen T, et al. Nasal continuous positive airway pressure reduces sleep-induced blood pressure increments in preeclampsia. Am J Respir Crit Care Med 2000; 162: 252-257.

43 Izci B, Riha RL, Martin SE, et al. The upper airway in pregnancy and pre-eclampsia. Am J Respir Crit Care Med 2003; 167: 137-140.

44 Ip MS, Lam B, Ng MM, et al. Obstructive sleep apnea is independently associated with insulin resistance. Am J Respir Crit Care Med 2002; 165: 670-676.

45 Okada M, Takamizawa A, Tsushima K, et al. Relationship between sleep-disordered breathing and lifestyle-related illnesses in subjects who have undergone health-screening. Intern Med 2006; 45: 891-896.

46 Davies GA, Maxwell C, McLeod L, et al. Obesity in pregnancy. J Obstet Gynaecol Can 2010; 32: 165-173.

47 Joel-Cohen SJ, Schoenfeld A. Fetal response to periodic sleep apnea: a new syndrome in obstetrics. Eur J Obstet Gynecol Reprod Biol 1978; 8: 77-81.

48 Yin TT, Williams N, Burton C, et al. Hypertension, fetal growth restriction and obstructive sleep apnoea in pregnancy. Eur J Obstet Gynecol Reprod Biol 2008; 141: 35-38. 